
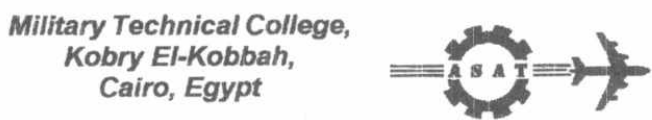

$9^{\text {th }}$ International Conference

On Aerospace Sciences \& Aviation Technology

\title{
LASER BEAM SCATTERING FROM CONDUCTING CIRCULAR CYLINDER COATED BY NEW SYNTHETIC ORGANIC POLYMERIC MATERIALS BASED ON POLY(ANILINE)
}

\author{
By \\ Fayed M.S. ", Aly M.S. ", Gouda S.R. ", Borham M.A. , \\ Azzam A.M., and Mohamed M. A."
}

\begin{abstract}
The problem of laser beam scattering from circular cylinder coated by organic dielectric polymeric material based on polyaniline is investigated. In this study, polyaniline is prepared in laboratory, it was chemically synthesized, via the chemical oxidation of aniline by persulfate anion in aqueous solution. It showed good yield and intermediate electrical conductivity $(\sigma=5 \mathrm{~S} / \mathrm{cm})$. This conductivity assigns it as a semiconductor material. Polyaniline was characterized by different methods of analysis such as: thermogravimetric analysis, electric conductivity, infrared and UV-visible absorption spectroscopy. The prepared polymer was formulated for laser beam scattering studies. The Gaussian beam scattering from an infinitely long conducting circular cylinder with large size parameter and located in the far zone from the source of excitation and coated by polyaniline dielectric material is investigated. This problem had been solved via modal expansion technique. The Gaussian beam is expanded in terms of continuous plane waves with amplitudes and phases given by a Gaussian weighting functions. The continuous plane wave spectrum of the Gaussian beam is obtained. Thus, the evaluation of the Gaussian beam scattering from the conducting circular cylinder reduces to evaluating the inverse Fourier transform to the scattered plane waves. Numerical results using a code developed under MATLAB environment is obtained. The obtained solution is compared with the previously published solutions that are based on modal expansion technique and good agreement is achieved. Experimental measurements are worked out for He-Ne laser beam of wave length $(\lambda=0.6328 \mu \mathrm{m})$ incident on conducting circular cylinder coated by different thickness of polyaniline material. The attenuation of the scattered field due to this dielectric material is computed and compared by the measured results.
\end{abstract}

\section{KEYWORDS}

Laser Beam; Laser Scattering; Conducting circular cylinder; Coated cylinder; Dielectric cylinder; Polyaniline; Organic conductor; Polymerization capacitance

"EGYPTIAN ARMED FORCES 


\section{INTRODUCTION}

Over the last decade or so there has been widespread interest in a new class of compounds known as conducting polymers. These polymers produce materials that combine the important electronic and optical properties of semiconductors and metals with specific mechanical properties. The organic polymeric conductors have attracted much scientific and technological interest in recent years. This is due to the large variety of recent and advanced applications in the electronic, photonic based devices and computer systems. The electrical conductivity of these polymers is considered to be intermediate between semiconductors and metals. It ranges between $1-1000 \mathrm{~s} / \mathrm{cm}$ [1]. These properties make them ideal candidates for use in : electronic, switching devices, rechargable batteries, sensors, corrosion inhibitors, capacitors which are used in all almost electronic equipments [2]. Exploitation of organic materials for electrochromic displays (ECDs) was done by several researches. More recently, the use of electroactive organic polymer has been tested widely. Polythiophene and polypyrrole and their analogues have been tested for ECDs use [3]. Currently commercial available capacitors are made of metalized inorganic film using metal oxide e.g., $\mathrm{M}_{\mathrm{n}} \mathrm{O}_{2}$ as cathodes. They have many disadvantages. They are subjected to severe thermal shock owing to high temperature typically $400-600^{\circ} \mathrm{C}$ required for the decomposition of manganese nitrate (III) during manufacture of the capacitor, also the capacitance is not constant beyond $10 \mathrm{kHz}$, and relatively low conductivity which limits the operation of the capacitor above $10 \mathrm{kHz}$. This enhances the failure rate of such capacitor in equipment assembly stage [4]. But using the conductive polymers solves these problems. Recently, conductive polymers have become one of the foci in material science research. This is due to their widespread technological applications. Also they used as an active electrode materials in energy storage [5], opto-electronic devices [6], display devices and were extensively searched to be from conducting polymers. These also may be used as chemically modified electrodes [7], and polymer temperature sensors [8]. In addition to the above applications, research and development are continuing in several areas in antistatic applications, sensors, radar blocking applications., and recently as actuators that do work similar to mechanicalelectrical micro machines (MEMs) [9]. In the present work, polyaniline was selected to be chemically prepared via its oxidation by ammonium Persulfate in an acid medium. The prepared polymer was analyzed and investigated to explore its chemical and electrical properties that can be useful in electronic applications as laser scattering and electromagnetic interference shieiding (EMIS). The formulation of the chemically synthesized conducting polyaniline was focused on conducting cylinders which represent one of the most important classes of scatterers. Many practical scatterers, such as fuselage of airplanes, pipelines, and missiles can be represented by cylindrical structures. Therefore, Gaussian beam scattering from conducting circular cylinder becomes the matter of interest for many researches and applications[14,15,16 ]. In most publications, the solution of the scattering problem is one of the main subjects in the electromagnetic theory[17 ]. The scattered field could be obtained using various techniques[15,18 ]. In this paper, the modal expansion technique is employed to solve the problem of conducting cylinder coated by dielectric cylinder based on polyaniline. The incident Gaussian beam is expanded in terms of plane wave constituents $[14,15,19]$. Then, applying the boundary condition for each constituent to get the scattered field from Gaussian beam. The scattered field due to Gaussian beam excitation is thus obtained by summing up the scattered field due to the incident plane waves spectrum[ $[4,19,20]$. 
For comparing the theoretical results with the experimental work, a practical setup is developed to measure the scattering pattern of $\mathrm{He}-\mathrm{Ne}$ laser beam incident on a perfectly conducting circular cylinder of radius $\mathrm{a}=4.5 \mathrm{~mm}$ that coated by polayaniline layer of different thickneses ranging from $0.18-0.23 \mathrm{~mm}$. The circular cylinder is located at distance $d=30 \mathrm{~cm}$ from the photodiode detector. An excellent agreement between the theoretical and practical results is obtained.

\section{EXPERIMENTAL WORK}

\subsection{Polymer Synthesis}

Polyaniline was chemically polymerized by the standard method of chemical oxidation with ammonium persulphate in the presence of hydrochloric acid as follows: Ammonium Persulfate was dissolved in hydrochloric acid and the solution was cooled to $0{ }^{0} \mathrm{C}$ using ice bath. aniline monomer was dissolved in hydrochloric acid The mixture was stirred for 15 minutes to insure complete solubility and was also cooled to $0^{\circ} \mathrm{C}$. Ferrous sulfate aqueous solution were added to the aniline solution. The ammonium Persulfate solution was gradually added to the aniline solution all over a period of 15 minutes while cooling. After one hour of vigorous stirring of the cold mixture, it was transferred to a freezer for about two hours, a dark brown precipitate was formed, filtered, washed and dried in an oven at $50^{\circ} \mathrm{C}$ for 5 days .

\subsection{Polymer Characterization}

Infrared absorption spectrum was carried out by dispersing samples of the product in $\mathrm{KBr}$ and measured on Perkin-Elmer model 3087 Spectrophotometer, in a wave number range of $500-4000 \mathrm{~cm}^{-1}$. The visible- UV absorption spectrum of the polymer solution was determined using UV-visible Shimmadzu NIR -160 spectrophotometer (Japan). Thermogravimetric analysis (TGA) of the product was carried out with a rate of $15{ }^{\circ} \mathrm{C}$ per minute, the test was conducted in the temperature range of $0-450^{\circ} \mathrm{C}$ using NETZSCH TG ZOQ instrument (west Germany). The electrical conductivity was determined using the electronic four point probe Multimeter model 120 Hewlett Packard (France). The test was carried out on a glass substrate with squared shape.

Polyaniline was synthesized by the standard method of chemical polymerization of aniline [10], via its oxidation by ammonium persulfate in $\mathrm{HCl}$ aqueous solution. In the present polymerization, ammonium persulfate was selected as oxidant, because it was found that it gives a high yield in aniline oxidation [11]. The weight of polyaniline product is quite high. The proposed structure of polyaniline includes quiniod and benzeniod moities in the backbone. The ratios of these two structures depend on the oxidation state occurred. The color was also related to the nature of quinione imine in the backbone. On this base the structure may be significantly modified by doping processes, which involve the oxidation or reduction of the polymer chain. Consequently, the electronic conductivity level, controlled synthesis and the other factors are the main parameters in these processes. As a result of these chain modification, the polymer can find unique application in advanced technologies. The proposed structure of polyaniline as a complete reduction, form 1, and a complete oxidized, form 2, imine and amine protonated forms are given as follows: 


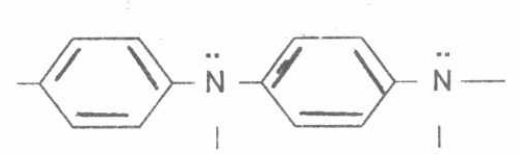

$\mathrm{H}$
$\mathrm{H}$<smiles>CCCCCCCCc1ccc(C)cc1</smiles>

$\mathbf{N}$

A. $N+$

$\mathrm{H}$

(1B)

FORM (1)<smiles>[X][V]Nc1ccc(N=C2C=CC(=C)C=C2)cc1</smiles>

$(2 \mathrm{~A})$

(2B)

FORM (2)

The possible mechanism of aniline polymerization is the loss of an electron from the lone pair to form the corresponding radical cation, which can be represented by the equation :

$$
\mathrm{C}_{6} \mathrm{H}_{4}-\mathrm{NH}_{2} \stackrel{\text { Oxidation }}{\longrightarrow} \quad \mathrm{C}_{6} \mathrm{H}_{4}-\stackrel{+}{\mathrm{N}} \mathrm{H}_{2}
$$

This radical can attack another molecule of aniline at the para-position. During this attack some structure modification occurs via migration of methoxy group to the o-position to form polyaniline. The degree of oxidation depends on the molar ratio of oxidizing agent, its time of addition and the type of catalyst used. The polymer weight obtained was $30.4 \mathrm{~g}$, which amounts to $85 \%$ yield. The polymer solubility was tested in different organic solvents. It was found that it is partially soluble in formamide, soluble in dimethyl formamide DMF, and completely insoluble in benzene, ethanol, methanol, and acetone. The electrical resistivity was measured by four point probe multimeter, and electrical conductivity was calculated according to the formula:

$$
\sigma=R D d / L
$$

where,

$\sigma$....... Conductivity of the polymer film $(\mathrm{S} / \mathrm{cm})$

$R$....... Resistivity (ohm).

$D$....... Height of the sample.

$L \ldots \ldots$. Separation between two silver base poles.

$d$...... Thickness of the polymer film $(\mu \mathrm{m})$. 
The electrical conductivity was amounted to $0.56,1.234,1.651 \mathrm{~S} / \mathrm{cm}$ corresponding to three samples prepared with different molar ratios (monomer/oxidant): $1: 1,1: 2$, and $1: 3$ respectively, and using persulfate in $1 \mathrm{M}$ aquéeous hydrochloric acid .

This behavior of polyaniline conductivity can be attributed to several factors such as: chemical structure of the polymer and the extent of oxidation. The measured conductivity lies in the semiconductor region. Thus, this polymer may be used as a semiconductor material in electronic devices. The role of hydrochloric acid, is the protonation of poly( $p$-aniline) to form the corresponding salt, this occurs preferentially at the imine nitrogen $(-\mathrm{N}=)$ as follows:

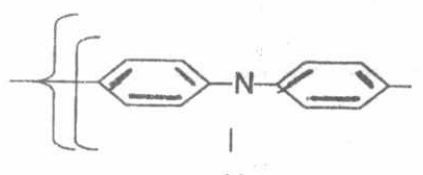

$\mathrm{H}$
$\mathrm{H}$

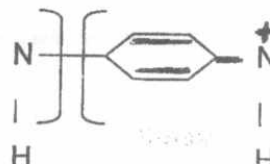

$\mathrm{H}$

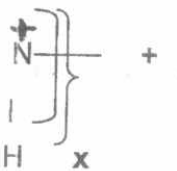

H x<smiles>CCc1ccc(Nc2ccccc2)cc1</smiles>

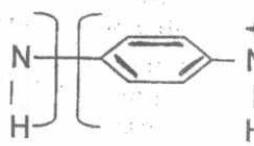<smiles>[X]c1ccc([NH+]=NC=CC2CC2)cc1</smiles>

$(2 \mathrm{~A}) \times$

This protonation reaction induce a large increase in the resultant polymer conductivity, since it is known that the totally oxidized form is an insulator, this doping process leads to the formation of a positive charge localized in the polymer chain accompanied by the transformation of aromatic structure towards the quiniod form. Such conductivity can be looked as a result of both the conjugation and regularity of the polymer structure. In the same time the oxidation implies the diffusion of the oppositely charged chloride counterions from the medium to the polymer.

\subsubsection{IR-spectrum of the polymer}

sample was tested and showed in Fig. 1. This spectrum reveals the existance of the bands in the region of $1400-1240 \mathrm{~cm}^{-1}$ which correspond to the $\mathrm{C}-\mathrm{N}$ stretching characteristics of aromatic amines. The peak at $1160 \mathrm{~cm}^{-1}$ corresponds to $\mathrm{C}=\mathrm{N}$ in the quinione imine moity. The aromaticity of the polymer can be shown by peaks at 745 $\mathrm{cm}^{-1}$ and $695 \mathrm{~cm}^{-1}$. The strong band at about $3500 \mathrm{~cm}^{-1}$ region may be attributed to the $\mathrm{N}-\mathrm{H}$ stretching. 




Fig. 1 IR - Spectrum of Polyaniline sample

\subsubsection{UV-visible spectrometry}

It was reported (5) that UV-visible spectra of the fully reduced form of the parent polymer, polyaniline, lecoemeriadine in N-methyl pyrolidone NMP reveals a strong absorption band centered at $340 \mathrm{~nm}$ and a very weak residual absorption at $633 \mathrm{~nm}$. The former band has been assigned to the transition corresponding to the benzeniod band ( $\mathrm{B}$-band). The latter band has been explained to be due to a charge-transfer excitation-like transition from the highest occupied level centered on the benzeniod rings to the lowest unoccupied energy level centered on quiniod rings. It is believed that this excitation band can be used as a measure of the oxidation state of polyaniline. As the intensity of excitation band decreases relative to $B$ band, the Oxidation State is probably low. The UV-visible spectrum of poly(aniline) as given in Fig. 2 shows characteristic bands at $315 \mathrm{~nm}$ and $350 \mathrm{~nm}$. The substitution on benzene rings of methoxy group causes a hypschromic shift of the transition band from 320 $\mathrm{nm}$ to $315 \mathrm{~nm}$. This band is related to the extent of the conjugation between the adjacent rings in the polymer chain. The resonance energy is at its maximum when the rings are coplanar. So the main effect of the substituent groups of methoxy on the rings, is the destruction of that coplanarity by the steric interference leading to the formation of the stable conformation with certain angle between the planes of the rings. By this way the maximum overlap can not be achieved. The band at $620 \mathrm{~nm}$ in polyaniline disappears in the spectrum of the salt and new peak at $420 \mathrm{~nm}$ was formed. This band was to be due to the charge transfer complex formed that absorb s in the visible region. This band is also shifted to $350 \mathrm{~nm}$ due to the same reason mentioned before. The reported results inspite of its rarity showed that the position of excition band is dependant on the chain length (degree of polymerization) as well as on the distribution of benzeniod and quiniod rings (extent of doping). The slight difference in absorption bands may be due to the extent of the oxidation and the used solvent. 


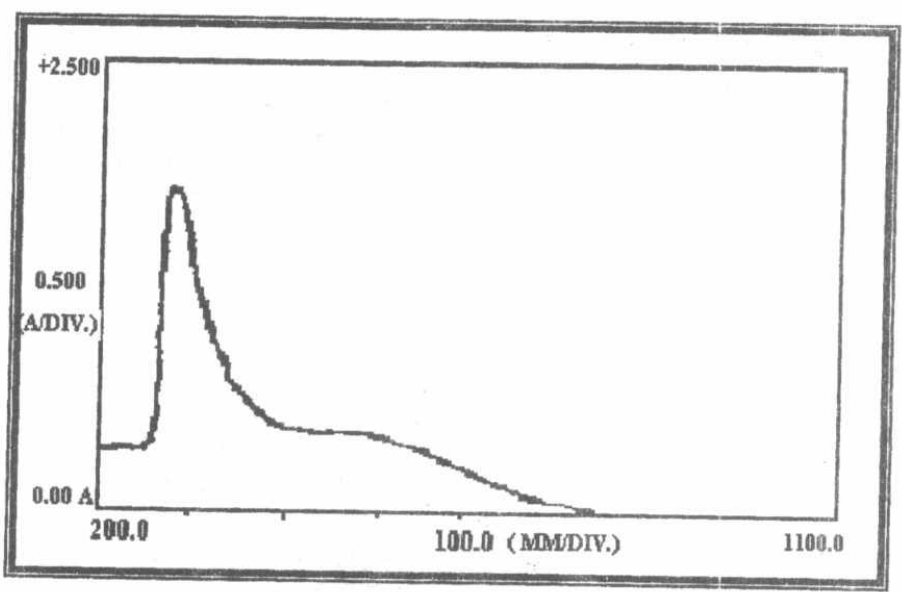

Fig. 2 UV-Visible absorption of polyaniline sample dissolved in formaınide

\subsubsection{Thermogravimetric Analysis (TGA)}

TGA (Fig.3) for the sample prepared at molar ratio 1:5 (monomer/oxidant) was tested. The polymer shows one weight loss region at $100^{\circ} \mathrm{C}$ with $37.8 \%$ loss in weight. This can be attributed to the removal of water. The second weight loss that was located at $295^{\circ} \mathrm{C}$, can be explained by the partial decomposition of the organic polymer. This thermal analysis indicates that the sample is stable up to $290^{\circ} \mathrm{C}$.

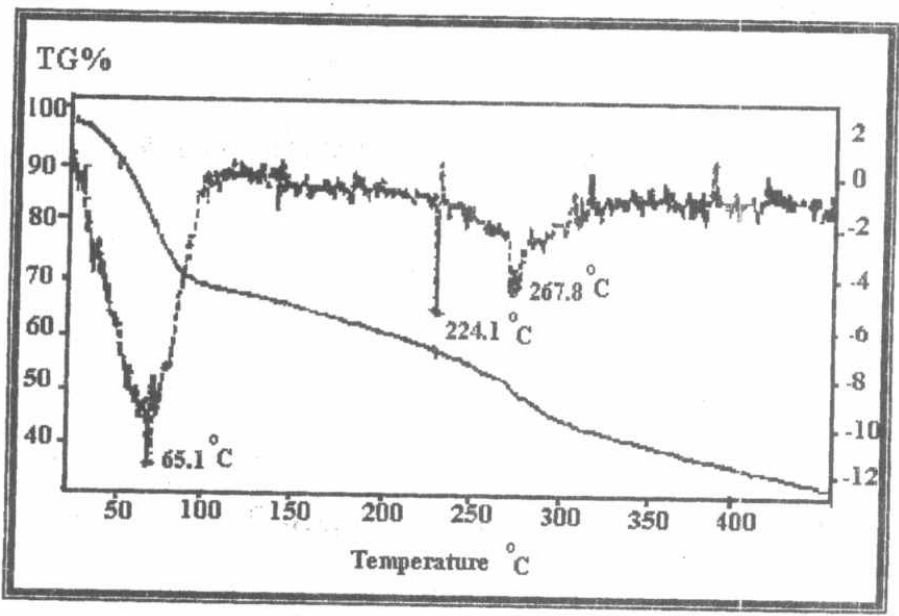

Fig. 3 Thermal-gravimetric data of polyaniline sample of one dynaic segment at $15{ }^{\circ} \mathrm{C}$ per min. 


\section{GEOMETRY OF THE PROBLEM}

The geometry of the problem is shown in Fig.4. An infinitely long perfectly conducting circular cylinder of radius " $a$ " coated by dielectric circular cylinder of radius " $b$ " placed at the origin are excited by a TM laser beam located along the negative $z$-axis at a distance $z_{\mathrm{o}}$ from the origin.

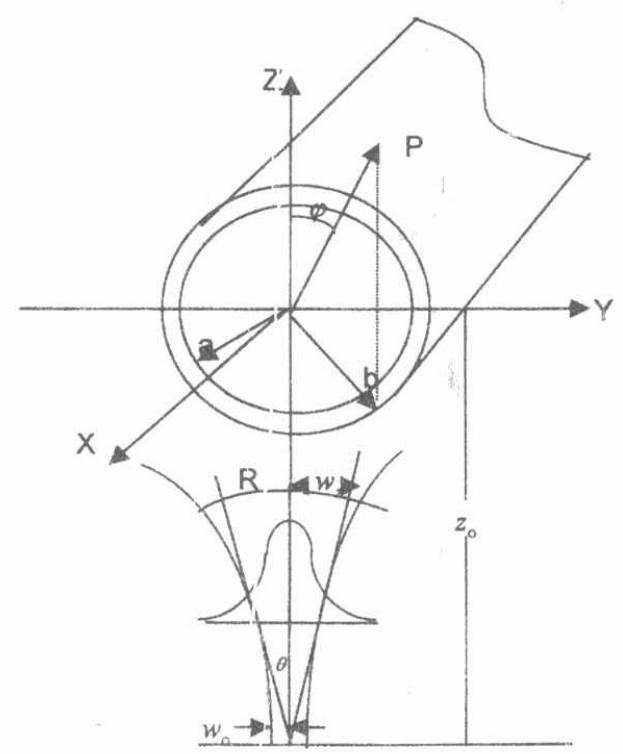

Fig.4 Schematic diagram of the incident Gaussian beam on a system of infinitely long perfectly conducting circular cylinder of radius "a" coated by dielectric cylinder of radius "b"

The system of equations for the electric field in that case are given as follows:

$$
\begin{aligned}
& E_{z}{ }^{i}=\hat{a}_{z} E_{\mathrm{o}} \sum_{n=-\infty}^{n=\infty} i^{-n} A_{n} J_{n}\left(k_{\mathrm{o}} \rho\right) e^{i n \phi} \\
& E_{z}{ }^{s}=\hat{a}_{z} E_{\mathrm{o}} \sum_{n=-\infty}^{n=\infty} a_{n} H_{n}^{(2)}\left(k_{\mathrm{o}} \rho\right) e^{i n \phi} \\
& E_{z}{ }^{d}=\hat{a}_{z} E_{\mathrm{o}} \sum_{n=-\infty}^{n=\infty}\left(b_{n} J_{n}\left(k_{1} \rho\right)+c_{n} Y_{n}\left(k_{1} \rho\right)\right) e^{i n \phi}
\end{aligned}
$$


Also, the corresponding magnetic field can be obtained by using Faraday equation, which in these instances reduces to:

$$
\begin{aligned}
& H_{z}^{i}=-\frac{1}{i \omega \mu_{\mathrm{o}}} \nabla \times E_{z}^{i} \\
& H_{z}^{s}=-\frac{1}{i \omega \mu_{\mathrm{o}}} \nabla \times E_{z}^{s} \\
& H_{z}^{d}=-\frac{1}{i \omega \mu_{1}} \nabla \times E_{z}^{d}
\end{aligned}
$$

Thus,

$$
\begin{aligned}
& H_{\phi}^{i}=-\frac{1}{i \omega \mu_{\mathrm{o}}} \frac{1}{\rho} \frac{\partial E_{z}^{i}}{\partial \rho} \\
& H_{\phi}^{i}=-\frac{1}{i \omega \mu_{\mathrm{o}}} \frac{1}{\rho} E_{\mathrm{o}} k_{\mathrm{o}} \sum_{n=-\infty}^{\infty} i^{-n} A_{n} J_{n}^{\prime}\left(k_{\mathrm{o}} \rho\right) e^{m \phi} \\
& H_{\phi}^{s}=-\frac{1}{i \omega \mu_{\mathrm{o}}} \frac{1}{\rho} \frac{\partial E_{z}^{s}}{\partial \rho} \\
& H_{\phi}^{s}=-\frac{1}{i \omega \mu_{\mathrm{o}}} \frac{1}{\rho} E_{\mathrm{o}} k_{\mathrm{o}} \sum_{n=-\infty}^{\infty} i^{-n} a_{n} H_{n}^{(2)^{\prime}}\left(k_{\mathrm{o}} \rho\right) e^{i n \phi} \\
& H_{\phi}^{d}=-\frac{1}{i \omega \mu_{1}} \frac{1}{\rho} \frac{\partial E_{z}^{d}}{\partial \rho} \\
& H_{\phi}^{d}=-\frac{1}{i \omega \mu_{1}} \frac{1}{\rho} E_{\mathrm{o}} k_{1} \sum_{n=-\infty}^{\infty} i^{-n}\left(b_{n} J_{n}^{\prime}\left(k_{1} \rho\right)+c_{n} Y_{n}^{\prime}\left(k_{1} \rho\right)\right) e^{i n \phi}
\end{aligned}
$$

\subsection{Solution of the scattering problem via modal Expansion}

The boundary conditions on the surface of the conducting cylinder will be:

$$
E_{z}^{d}=0 \quad \rho=a, \quad 0 \leq \phi \leq 2 \pi
$$

The boundary conditions on the surface of the dielectric cylinder will be:

$$
E_{z}^{i}+E_{z}^{s}=E_{z}^{d} \quad \rho=b \quad 0 \leq \phi \leq 2 \pi
$$




$$
H_{z}^{i}+H_{z}^{s}=H_{z}^{d} \quad \rho=b \quad 0 \leq \phi \leq 2 \pi
$$

Applying the boundary condition we get:

At $\rho=a, 0 \leq \phi \leq 2 \pi$

$$
E_{z}{ }^{d}=\hat{a}_{z} E_{\mathrm{o}} \sum_{n=-\infty}^{n=\infty}\left(b_{n} J_{n}\left(k_{1} \rho\right)+c_{n} Y_{n}\left(k_{1} \rho\right)\right) e^{i n \phi}=0
$$

$$
b_{n} J_{n}\left(k_{1} \rho\right)+c_{n} Y_{n}\left(k_{1} \rho\right)=0
$$

$c_{n}=-b_{n} \frac{J_{n}\left(\beta_{1} a\right)}{Y_{n}\left(\beta_{1} a\right)}$

At

$\rho=b ; 0 \leq \phi \leq 2 \pi$

$$
E_{z}^{i}+E_{z}^{s}=E_{z}^{d}
$$

$$
H_{z}^{i}+H_{z}^{s}=H_{z}^{d}
$$

$$
\begin{aligned}
& b_{n} J_{n}\left(\beta_{1} b\right)+c_{n} Y_{n}\left(\beta_{1} b\right)=j^{-n} J_{n}\left(\beta_{0} b\right)+a_{n} H_{n}^{(2)}\left(\beta_{0} b\right) \\
& b_{n}\left[J_{n}\left(\beta_{1} b\right)-\frac{J_{n}\left(\beta_{1} b\right) Y_{n}\left(\beta_{1} b\right)}{Y_{n}\left(\beta_{1} a\right)}\right]-a_{n} H_{n}^{(2)}\left(\beta_{0} b\right)=j^{-n} J_{n}\left(\beta_{0} b\right)
\end{aligned}
$$

And

$\frac{\beta_{1}}{\mu_{n}} b_{n}\left[J_{n}^{\prime}\left(\beta_{1} b\right)-\frac{J_{n}\left(\beta_{1} b\right) Y_{n}^{\prime}\left(\beta_{1} b\right)}{Y_{n}\left(\beta_{1} a\right)}\right]-a_{n} H_{n}^{(2)}\left(\beta_{0} b\right)=j^{-n} \frac{\beta_{0}}{\mu_{0}} J_{n}^{\prime}\left(\beta_{0} b\right)$

Rearranging the coefficient we get the matrix:

$\left[\begin{array}{cc}-H_{n}^{(2)}\left(\beta_{0} b\right) & \frac{J_{n}\left(\beta_{1} b\right) Y_{n}\left(\beta_{1} a\right)-J_{n}\left(\beta_{1} a\right) Y_{n}\left(\beta_{1} b\right)}{Y_{n}\left(\beta_{1} a\right)} \\ -\frac{\beta_{0}}{\mu_{0}} H_{n}^{(2)}\left(\beta_{0} b\right) & \frac{\beta_{1}}{\mu_{1}} \cdot \frac{J_{n}^{\prime}\left(\beta_{1} b\right) Y_{n}\left(\beta_{1} a\right)-J_{n}\left(\beta_{1} a\right) Y_{n}^{\prime}\left(\beta_{1} b\right)}{Y_{n}\left(\beta_{1} a\right)}\end{array}\right]\left[\begin{array}{l}a_{n} \\ b_{n}\end{array}\right]=\left[\begin{array}{c}j^{-n} J_{n}\left(\beta_{0} b\right) \\ j^{-n} \frac{\beta_{0}}{\mu_{0}} J_{n}^{\prime}\left(\beta_{0} b\right)\end{array}\right]$

The previous $(2 \times 2)$ matrix equation is obtained based on the coupled boundary condition expressions

$$
[\mathrm{M}][\mathrm{A}]=[\mathrm{V}]
$$


Once the unknown coefficients $a_{n}$ and $b_{n},[\mathrm{~A}]$, are known, the components of the electric field and the magnetic field can be calculated.

Thus;

$$
a_{n}=\frac{a_{n 1}-a_{n 2}}{a_{n 3}-a_{n 4}}
$$

where,

$$
\begin{aligned}
& a_{n 1}=j^{-n} \sqrt{\frac{\varepsilon_{r}}{\mu_{r}}} \cdot J_{n}\left(\beta_{o} b\right) \frac{J_{n}^{\prime}\left(\beta_{1} b\right) Y_{n}\left(\beta_{1} a\right)-J_{n}\left(\beta_{1} a\right) Y_{n}^{\prime}\left(\beta_{1} b\right)}{Y_{n}\left(\beta_{1} a\right)} \\
& a_{n 2}=j^{-n} J_{n}^{\prime}\left(\beta_{o} b\right) \frac{J_{n}\left(\beta_{1} b\right) Y_{n}\left(\beta_{1} a\right)-J_{n}\left(\beta_{1} a\right) Y_{n}\left(\beta_{1} b\right)}{Y_{n}\left(\beta_{1} a\right)} \\
& a_{n 3}=-\sqrt{\frac{\varepsilon_{r}}{\mu_{r}}} \cdot H_{n}^{(2)}\left(\beta_{0} b\right) \frac{J_{n}^{\prime}\left(\beta_{1} b\right) Y_{n}\left(\beta_{1} a\right)-J_{n}\left(\beta_{1} a\right) Y_{n}^{\prime}\left(\beta_{1} b\right)}{Y_{n}\left(\beta_{1} a\right)} \\
& a_{n 4}=-H_{n}^{(2)}\left(\beta_{0}^{\prime} b\right) \frac{J_{n}\left(\beta_{1} b\right) Y_{n}\left(\beta_{1} a\right)-J_{n}\left(\beta_{1} a\right) Y_{n}\left(\beta_{1} b\right)}{Y_{n}\left(\beta_{1} a\right)}
\end{aligned}
$$

As the same for the unknown coefficient $b_{n}$ :-

$$
b_{n}=\frac{b_{n 1}-b_{n 2}}{b_{n 3}-b_{n 4}}
$$

where,

$$
\begin{aligned}
& b_{n 1}=-j^{-n} J_{n}^{\prime}\left(\beta_{0} b\right) H_{n}^{(2)}\left(\beta_{0} b\right) \\
& b_{n 2}=-j^{-n} J_{n}\left(\beta_{0} b\right) H_{n}^{(2)^{\prime}}\left(\beta_{\mathrm{o}} b\right) \\
& \left.b_{n 3}=j^{-n} \sqrt{\frac{\varepsilon_{r}}{\mu_{r}}} \cdot H_{n}^{(2)} \beta_{\mathrm{o}} b\right) \frac{J_{n}^{\prime}\left(\beta_{1} b\right) Y_{n}\left(\beta_{1} a\right)-J_{n}\left(\beta_{1} a\right) Y_{n}^{\prime}\left(\beta_{1} b\right)}{Y_{n}\left(\beta_{1} a\right)}, \\
& \left.b_{n 4}=j^{-n} \sqrt{\frac{\varepsilon_{r}}{\mu_{r}}} \cdot H_{n}^{(2)} \beta_{0} b\right) \frac{J_{n}\left(\beta_{1} b\right) Y_{n}\left(\beta_{1} a\right)-J_{n}\left(\beta_{1} a\right) Y_{n}\left(\beta_{1} b\right)}{Y_{n}\left(\beta_{1} a\right)},
\end{aligned}
$$




\subsection{LASER BEAM SCATTERING MEASUREMENTS}

The laser beam scattering measurements were carried on four identical circular metallic cylinders of different coating thickness. The measuring system contained the following devices: UNIPHASE He-Ne laser source model 1101P of wavelength $(\lambda=$ $0.6328 \mu \mathrm{m}$ ) with power $4 \mathrm{~mW}$, beam diameter of $0.63 \mathrm{~mm}$, and beam divergence of $1.5 \mathrm{mrad}$. Power meter and goniometer for measuring the angle of the reflected bearn. The configuration of this system is shown in Fig.5. The data obtained on scattering were employed to compare the experimental results with numerical results that computed via codes based on MATLAB environment.

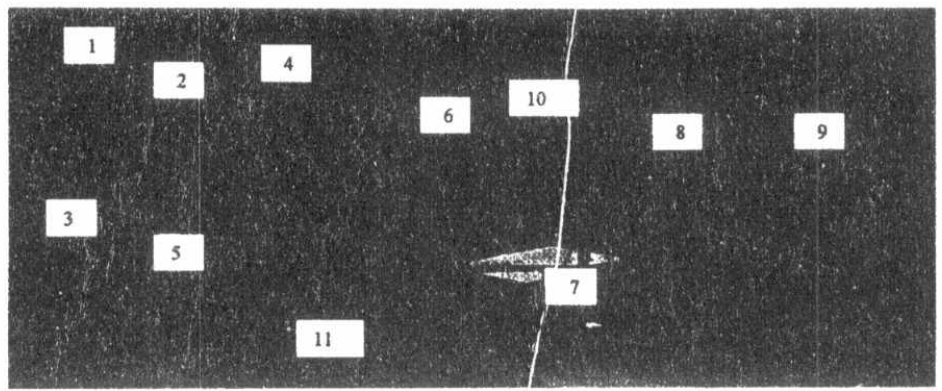

Fig5. Experimental setup of $\mathrm{He} \_\mathrm{Ne}$ laser scattering by a conducting circular cylinder

(1) He_Ne laser $(\lambda=0.6328 \mu \mathrm{m})$; output power $=4 \mathrm{~mW}$.

(2) Driver of He_Ne laser ( ON / OFF switch).

(3) Chopper

(4) Driver of chopper

(5) Iris

(6) Si detector $(0.4-1.1 \mu \mathrm{m})$ with focusing lens.

(7) Conducting circular cylinder

(8) Detector power meter

(9) Oscilloscope $(50 \mathrm{MHz})$

(10) goniometer

(11) Optical bench 


\subsection{ANALYSIS AND DISCUSSION}

Figure 6 shows a code verification for the incident field $E_{i}$, scattered field $E_{s}$, and transmitted field $E_{d}$ of the Gaussian beam incident on a conducting circular cylinder of size parameter $x=2 \pi a / \lambda=10$ and spot size to radius ratio $\mathrm{wz} / \mathrm{a}=1$ coated with dielectric cylinder.

Figures $(7-11)$ report a He-Ne laser scattering pattern from both metallic circular cylinder of diameter $(\mathrm{d}=9 \mathrm{~mm})$ and metallic circular cylinders of the same diameter but coated with different thickness of thin film made from polymeric dielectric material based on polyanialine. The laser beam has an incident power $\mathrm{P}_{\text {inc }}=4 \mathrm{~mW}$, wavelength $(\lambda=0.6328 \mu \mathrm{m})$, and is operating in TEMOO mode with vertical polarization. It is located at a distance $4.5 \mathrm{~m}$ from the scatterer as shown in Fig. 5.

Fig. 7 shows the comparison between the measured and calculated scattered field from non-coated cylinder. The degree of coincidence of both results is reasonable in view of the tolerance and accuracy involved in the scatterer fabrication. The calculation is made via a developed MATLAB code. It is noted that the scattering pattern is directive in the backward direction and has no side lobe.

Fig. 8 shows the measured scattering pattern from a coated cylinder where the coated depth is $0.18 \mathrm{~mm}$. It is observed that the peak-detected power relative to the incident power is $0.95 \times 10^{-6}$ thus, the peak-detected power for this coated case is reduced by $96 \%$. Increasing the coating depth from $0.18 \mathrm{~mm}-0.25 \mathrm{~mm}$, at figures $9-11$, increase the reduction ratio by only $1 \%$. In the mean time the scattering pattern shows no enhanced dispersion relative to the non-coated cylinder. In view of the above observation, it is clear that the employed material is highly dissipative and the depth increase from $0.18-0.25 \mathrm{~mm}$ does not highly affect the results in all cases.

\section{CONCLUSION}

Polyaniline was synthesized successfully by the chemical polymerization of aniline. Via its oxidation by ammonium persulfate in aqueous solution, by employing ferrous sulfate as a catalyst. The characterization of the prepared polymer was done by UV- IR spectroscopy, thermogravimetric analysis, solubility and electrical conductivity, The resultant electrical conductivity was in the range $0.54-1.65 \mathrm{~s} / \mathrm{cm}$, making it very useful as a semiconductor material. It is clear, from the TG-analysis, that the polymer sample is stable up to $290^{\circ} \mathrm{C}$. Due to this thermal stability, it may be find suitable applications in semiconductor devices. Polyaniline proved that it is comparable to the currently used non-polymeric material in addition to the better mechanical, electrical, and thermal properties thus it can be find several applications. The polyaniline can be used as a coating of conducting surfaces of cylindrical shapes to reduce the amplitude of the reflected He-Ne laser beam. It was found that the coating thickness around $0.2 \mathrm{~mm}$ might be considered as the suitable thickness to perform the desired
attenuation. 


\section{RFERENCES}

[1] M.Goosey, (1987), Speciality Polymers, $1^{\text {st }}$ ed. Chapman and Hall, New York, 85

[2] S.P.Sitaram, J.O.Stoffer, and T.J.O.Keefe, (1997), J of Coating Technology, 69 (688), 65.

[3] A.Kitani, Junyano and Kazuosaki, (1996), J of Electroanal.Chem., 209,227.

[4] S.Sathiyanarayanan et al, (1995), Syntheic Metals, 74, 165-170.

[5] T.A.Snothrem (ed.), ((1986), Handbook of conducting Polymers, Volume 1 and 2 , Marcel Decker. New york.

[6] Chao and M.S Wrighton, (1987), JAMC., 109, 6627.

[7] J.Josheph and D.C Trievedt, (1988), Bull.Electrochem., 4, 469.

[8] J Chem.Phys, 1990, 92 (4), 2187-88.

[9] Genies.E.M, Boyle A, Lapkoski M and T Sintavis.C, Syntheic Metals, 36, 139$82,1980$.

[10] S.Sathiyanarayanan et al, (1992), Corrosion Science, Volume 32 (12), 1831-44.

[11] J Aber, (1989), Science, 246, 208.

[12] M.S.Fayed, M.A.Radwan, , S.R.Gouda. M.A.Borham, (1999), 8 th international conference on aerospace science and aviation technology, 4-6 may, egypt.

[13] A. M. Azzam, M. S. Aly, Y. A. Badr, M. F. Hasan, and A. M. Elmahdy, (2000), "Gaussian beam scattering from very large conducting sphere with size comparable with beam footprint", Al-azhar Sixth International Engineering Conference, AlEC, September 1-4, Cairo, Egypt, pp. 266-282

[14] A. M. Azzam, Y. A. Badr, M. F. Hasan, M. S. Aly, and A. M. Elmahdy, (2001), "Far field scattering of Gaussian beam by an infinitely long conducting circular cylinder", 9th international conference on aerospace science and aviation technology, May 8-10, MTC, Cairo, Egypt. pp.

[15] A. Z. Elsherbeni and et al., (1993), Iterative scattering of a Gaussian bezam by an array of circular conducting and dielectric cylinders, Journal of Electromagnetic waves and applications, Vol. 7, No. 10, P.P $1323-1342$.

[16] A. Z. Elsherbeni and A. Kishk, (1992), Modeling of cylindrical objects by circular dielectric or conducting cylinders, IEEE Trans. Antennas Propagat., Vol. AP-40, P.P 96-99.

[17] Balanis,C.A., (1989), Advanced Engineering Electromagnetics, New York Wiely.

[18] Harrington, R.F., (1968), Field Computation By Moment Methods, MacMillan, New York.

[19] James A. Lock, (1995), Improved Gaussian beam-scattering algorithm," Applied Optics, Vol. 34, No. 3, P.P 559-570.

[20] James A. Lock, (1995), Interpretation of extinction in Gaussian-beam scattering, J'. Opt. Soc. Am. A., Vol. 12, No. 5, P.P 929-938. 


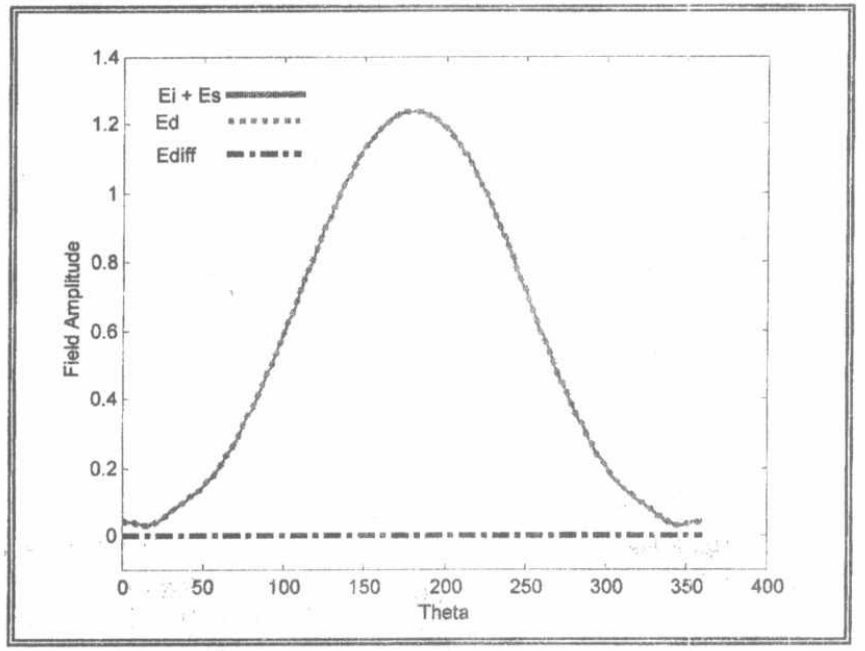

Fig. 6 Code verification for the incident field $E_{i}$, scattered field $E_{s}$, and transimitted field $\mathrm{E}_{\mathrm{d}}$ of the Gaussian beam incident on a conducting circular cylinder of size parameter $x=2 \pi a / 2=10$ and spot size to radius ratio $\mathrm{Wz} / \mathrm{a}=1$ coated with dielectric cylinder

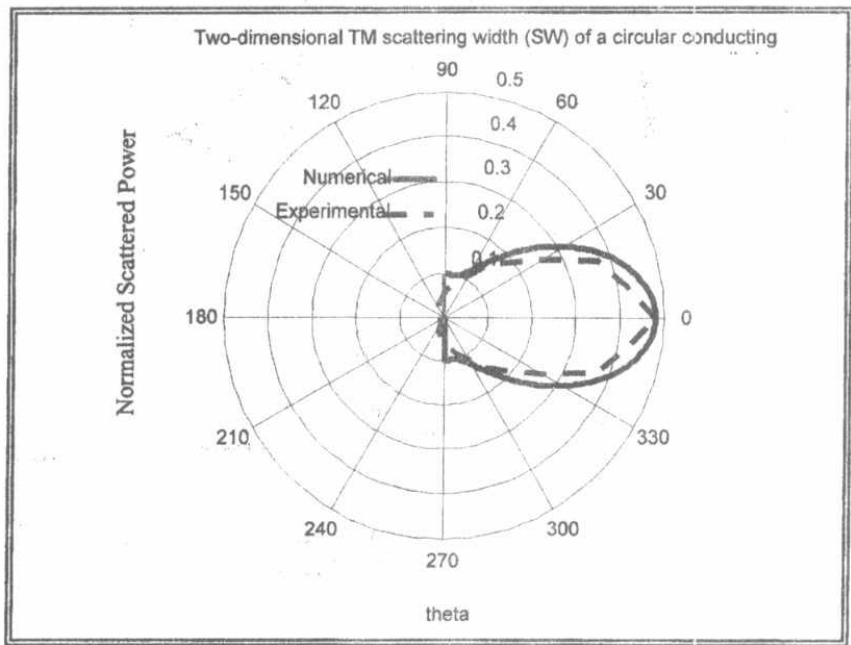

Fig. 7 Comparison between experimental \& numerical computation of $\mathrm{He}-\mathrm{Ne}$ laser scattering from conducting circular cylinder 


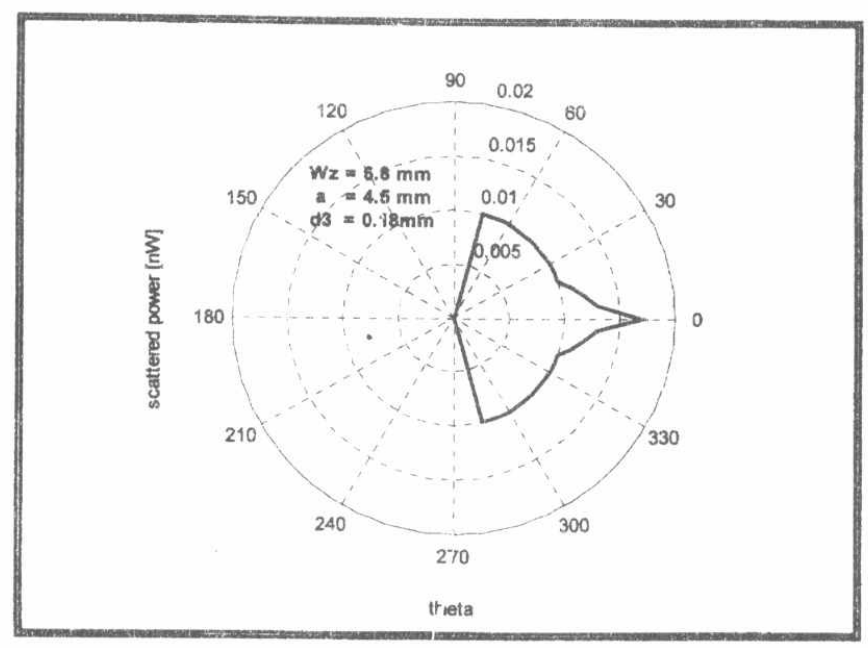

Fig. $8 \mathrm{He}-\mathrm{Ne}$ laser bearn $\left[\mathrm{W}_{\mathrm{z}}=6.8 \mathrm{~mm}\right]$ scattering from conducting circular cylinder of radius $[\mathrm{a}=4.5 \mathrm{~mm}]$ coated by polyanialine layer with thickness $(\mathrm{dl}=0.18 \mathrm{~mm})$

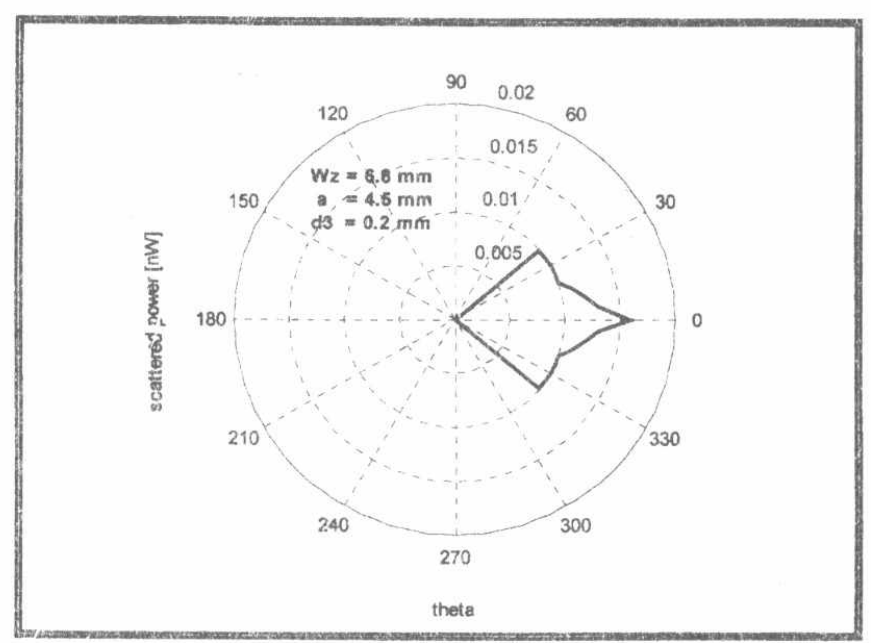

Fig. $9 \mathrm{He}-\mathrm{Ne}$ laser beam $\left[\mathrm{W}_{\mathrm{z}}=6.8 \mathrm{~mm}\right]$ scattering from conducting circular cylinder of radius $[a=4.5 \mathrm{~mm}]$ coated by polyanisidine layer with thickness $(\mathrm{d} 1=0.2 \mathrm{~mm})$ 


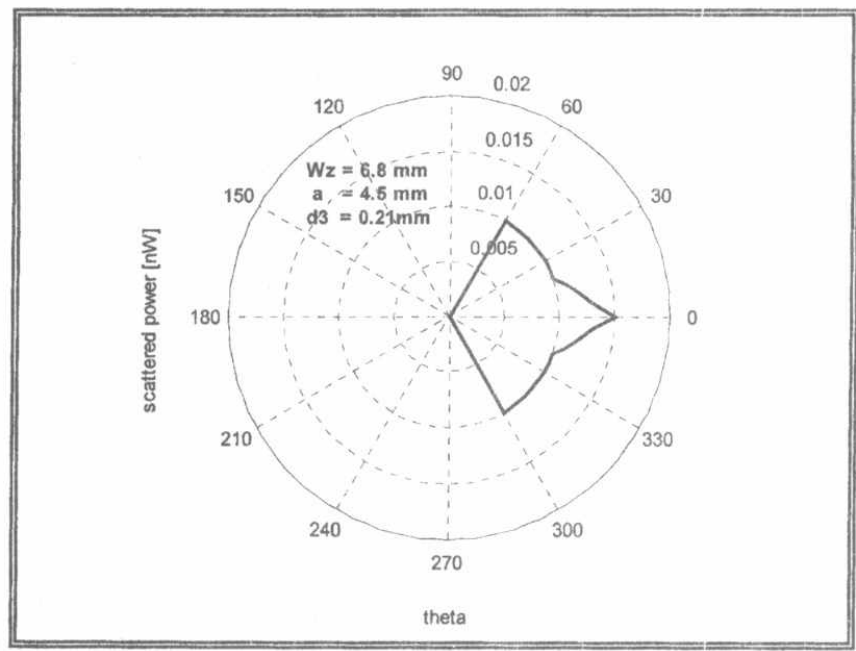

Fig. $10 \mathrm{He}-\mathrm{Ne}$ laser beam $\left[\mathrm{W}_{\mathrm{z}}=6.8 \mathrm{~mm}\right]$ scattering from conducting circular cylinder of radius $[\mathrm{a}=4.5 \mathrm{~mm}]$ coated by polyanialine layer with thickness $(\mathrm{d} 2=0.21 \mathrm{~mm})$

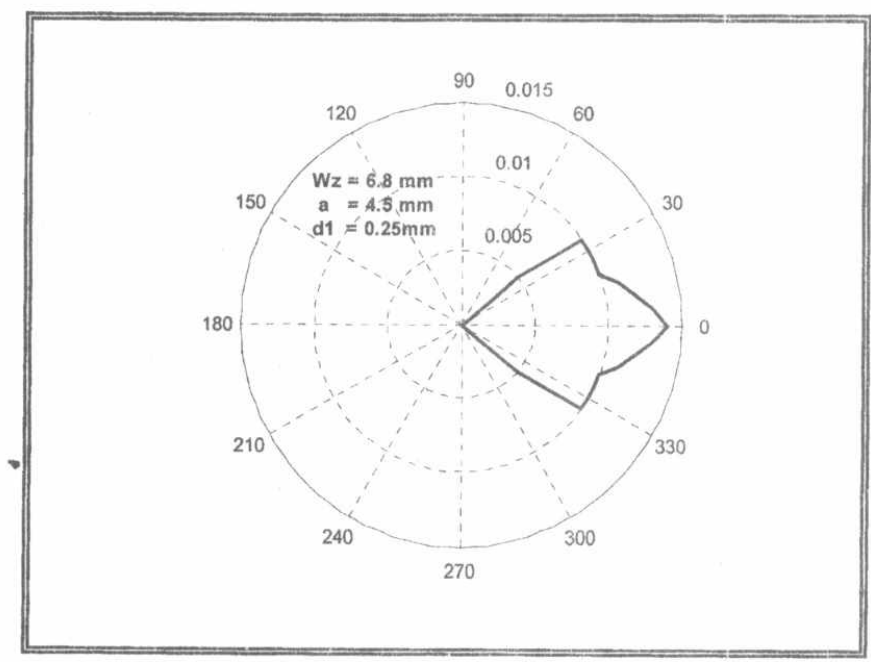

Fig. $11 \mathrm{He}-\mathrm{Ne}$ laser beam $\left[\mathrm{W}_{2}=6.8 \mathrm{~mm}\right]$ scattering from conducting circular cylinder of radius $[\mathrm{a}=4.5 \mathrm{~mm}]$ coated by polyanialine layer with thickness $(\mathrm{d} 1=0.25 \mathrm{~mm})$ 